\title{
The Severe End of the Spectrum: Insights and Opportunities from the Autism Inpatient Collection (AIC)
}

\author{
Matthew Siegel ${ }^{1}$ \\ Published online: 3 September 2018 \\ ○) Springer Science+Business Media, LLC, part of Springer Nature 2018
}

\begin{abstract}
Research on individuals severely affected by autism, including those who are minimally verbal, have intellectual disability or challenging behaviors, has become less common. The Autism Inpatient Collection (AIC) was initiated so data on this group is available to the research community. Ten studies utilizing phenotypic data from the first 350 AIC participants are presented. Greater autism severity, sleep disturbance, and psychiatric disorders are risks for hospitalization; fluently verbal youth experience more depression and oppositional symptoms; lower adaptive/coping skills are associated with increased problem behaviors; lower IQ is a risk for SIB; post-traumatic and suicidal symptoms are common; and challenging behaviors improve with specialized inpatient treatment. A new measure of emotion regulation and prescribing practices are described and future research discussed.
\end{abstract}

Keywords Autism $\cdot$ Inpatient $\cdot$ AIC $\cdot$ Verbal ability $\cdot$ Psychiatric

This special issue of the Journal of Autism and Developmental Disorders presents a series of ten papers that draw on phenotypic data from the first 350 participants in the autism inpatient collection (AIC), a growing database of over 1000 rigorously characterized psychiatrically hospitalized youth with ASD. By focusing on this sector of the ASD population, which is typically difficult to access, the investigators and authors have collectively sought to make information available on an under-researched group, and in some cases to challenge existing ideas about autism developed through studies of higher functioning samples. While there is not an agreed-upon definition of severity, or severe autism, in the field, the AIC contains a rich representation of those who are severely affected in one or more domains, including those who are minimally-verbal (52\%), have concurrent Intellectual Disability (43\%), and/or have serious challenging behaviors, such as physical aggression $(>90 \%)$ or selfinjury (22\%) (Siegel et al. 2015).

The AIC developed out of a recognition that youth severely affected by ASD are under-represented in the research literature and that a high volume of these youth

Matthew Siegel

siegem@mainebehavioralhealthcare.org

1 Maine Medical Center Research Institute, 509 Forest Avenue, Portland, ME 04101, USA were readily available for research recruitment through specialized child psychiatry units in the United States (Siegel et al. 2012). In 2013 six of these specialized units formed the Autism and Developmental Disorders Inpatient Research Collaborative, and engaged with scientific and philanthropic leaders in autism research to determine the best approach for utilizing this resource, from which grew the AIC study. Modeled after the highly successful outpatient Simons Simplex Collection, the AIC utilized a standardized assessment battery of measures valid for the full range of the autism spectrum, and a unique inpatient environment with $24 \mathrm{~h}$ access to participants for an average of 25.6 days each, to collect phenotypic data and biological samples (Siegel et al. 2015). De-identified phenotypic data have been made available to approved investigators as successively larger and more complete data sets through SFARI Base (http://www. sfari.org/resource/sfari-base/).

While not presented in this special issue, a primary reason for creating the AIC was to also make genetic sequencing data on this cohort available, thereby reducing the risk that conclusions drawn from current large collections of genetic data could be biased by overrepresentation of the higher functioning portion of the ASD population. The AIC data are anticipated to: (1) fill gaps in the autism genetic library, (2) facilitate high volume genomic interrogations of the full spectrum and accelerate the identification of autism 
subtypes, and (3) provide critical information to families affected by autism, particularly to those families affected by more severe forms of autism and having some of the greatest need. Sequencing data are anticipated to be available to approved investigators in the near future through SFARI Base.

\section{This Special Issue: Characteristics and Insights from the AIC}

This special issue features ten papers analyzing data from the Autism Inpatient Collection (AIC), a multi-site study of children and adolescents with ASD ages 4-20 years old who were admitted to one of six specialized inpatient psychiatry units. In some instances, comparison groups of ASD youth in outpatient treatment settings were also examined. In addition to contributing to our understanding of autism by utilizing the full spectrum, these papers also shed light on the inpatient ASD population and its treatment, which is critical given that approximately $11 \%$ of children with ASD are reported to be psychiatrically hospitalized before age 21 (Mandell 2008).

Predictors of inpatient psychiatric hospitalization were examined by Righi and colleagues using the AIC and a comparison cohort of age and sex matched children with ASD who had never been psychiatrically hospitalized (Righi et al. 2017). The authors examined three classes of predictors: individual characteristics, family resources, and the severity of psychiatric condition/behavioral presentation. The authors found that lower adaptive functioning, greater ASD symptom severity, having a single primary caregiver, the presence of a mood disorder, and sleep problems were all independent predictors of increased risk for psychiatric hospitalization. This study provides insight into which children with ASD may require this level of care in order to be safe and functional within their community.

Wink and colleagues completed the first study to prospectively capture psychotropic medication usage data in a large sample of ASD youth admitted to inpatient psychiatric facilities (Wink et al. 2017). Across the cohort, $91.7 \%$ were treated with at least one psychotropic medication, which is much higher than the 55-65\% rate described in most outpatient reports (Rosenberg et al. 2010). Antipsychotics were the most frequently employed class of medications, which is not surprising given that acute behavioral crisis typically precipitates admission. Relatively high rates of antidepressants, ADHD, or sleep medications underscore the prevalence with which these psychiatric comorbidities impact this population. Participants remained on a relatively stable number of psychotropic medications from admission to discharge, underscoring the behavioral severity, comorbidities, and treatment refractory nature of youth with ASD requiring inpatient admission. Interestingly, the percentage of patients on zero medications increased from $2.9 \%$ at discharge to $36 \% 2$ months post-discharge, which may point to a discrepancy between inpatient and community practitioner prescribing practices or to durability in the effect of inpatient behavioral treatment.

To examine effects of specialized inpatient treatment, an analysis of changes in problem behavior severity from admission to discharge and 2 months post-discharge was conducted by Pedersen and colleagues (Pedersen et al. 2017). Using the Aberrant Behavior Checklist-Irritability subscale, the authors found that serious problem behaviors (physical aggression, self-injury and severe tantrums) decreased from admission to discharge, and that improvement was sustained at 2 months post-discharge, though improvement in tantrum-like behaviors regressed slightly from discharge to the 2 month follow-up. Despite its disproportionately high significance for children with ASD, research on psychiatric hospital treatment has mostly emerged only in the past decade, with increasing attention toward the utilization of inpatient psychiatric services and the development and effectiveness of specialized inpatient programming.

Two studies in this special issue utilized the AIC's large amount of data on minimally verbal and intellectually disabled youth to deepen our understanding of the relationship between verbal ability and psychiatric symptom presentation and problem behavior. Lerner and colleagues compared children from the AIC with those recruited through an outpatient developmental disabilities clinic, to provide the first large scale estimate of differences in psychiatric symptom profiles between minimally verbal and fluently verbal youth with ASD, using the Child and Adolescent Symptom Inventory 5 (CASI-5) (Gadow 2013; Lerner et al. 2017). Both verbal ability groups were significantly impacted by psychiatric comorbidity as assessed by symptom severity. Fluently verbal youth with ASD were more likely to exceed clinical cut-offs for depression and oppositional defiant disorder, and had greater impairment due to depression symptoms compared to minimally verbal individuals. The finding that fluently verbal youth were more likely to experience depressive or oppositional symptoms was robust even after controlling for IQ, and prompts speculation that verbal youth may be at particular risk for experiences that produce depressive and oppositional reactions.

Examination of the relationship between verbal ability and problem behaviors in ASD has produced conflicting results, with some researchers reporting an inverse relationship, and others the opposite (Baghdadli et al. 2003; Matson et al. 2009). Using the AIC cohort, Williams et al. examined problem behaviors at hospital admission and found that both fluently verbal and minimally verbal youth exhibited a high severity and frequency of problem behaviors (Williams et al. 2017). Controlling for IQ and age, the verbal ability 
groups did not differ in their severity of SIB, stereotypy, or irritability on the Repetitive Behavior Scale-Revised, SIB subscale (RBSR-SIB) (Bodfish et al. 2000; Lam and Aman 2007) or the Aberrant Behavior Checklist-Irritability subscale (ABC-I) (Aman et al. 1985). Instead, increasing problem behavior severity was associated with lower adapting/ coping scores on the Vineland 3, even after controlling for verbal ability. Adaptive/coping behaviors require flexibility and differential responses to contextual demands, as well as cognitive control, regardless of verbal ability. This finding suggests the potential primacy of the relationship between problem behaviors and poor adapting/coping ability, distinct from verbal ability, and underscores the importance of developing adapting/coping strategies to address problem behaviors across the spectrum.

While it is well known that children with developmental disabilities are at higher risk for abuse (Hall-Lande et al. 2014), how an experience of trauma may present in youth with ASD is not well described. Brenner and colleagues utilized the AIC data to identify that a history of trauma was associated with more intrusive thoughts, distressing memories, loss of interest, irritability, and lethargy on the CASI-5 and ABC (Brenner et al. 2017). Individuals meeting clinical criteria for post-traumatic stress disorder (PTSD) scored higher on measures of externalizing behavior, suggesting that problem behaviors, which are often approached from a functional perspective in applied behavioral analysis (ABA), may in some instances also be symptomatic of trauma. A history of trauma was also unrelated to the severity of core symptoms of ASD, suggesting that less severe ASD core symptoms are not necessarily protective from traumatic experiences. This study revealed a high rate of parent-reported trauma for inpatient youth with ASD and described associated behavioral manifestations, underscoring the need for trauma screening and awareness of potential red flags in a child's behavior.

In a related vein, Horowitz and colleagues highlighted the prevalence of suicidality in verbal hospitalized children with ASD in the AIC, finding that $22 \%$ often or very often talked about death or suicide, by parent report (Horowitz et al. 2017). Importantly, talking about death or suicide was associated with meeting clinical criteria for co-morbid mood and anxiety disorders on the CASI-5, again suggesting that youth with ASD are not protected from serious problems, such as suicidality, when they experience anxiety or mood impairment. These findings indicate a critical need to develop valid and reliable suicide screening measures for this population.

Sannar et al. evaluated the relationship of behavioral presentation at admission and sleep during the hospitalization (2017). The authors found that greater irritability, stereotypy, and hyperactivity were associated with significantly fewer minutes of observed sleep during the last five nights of the hospital stay. The findings suggest that after adjusting to the hospital unit and when nearing readiness for discharge, a relationship between sleep and problem behavior emerges, raising the possibility of an etiology common to both sleep and behavior problems in ASD, such as neurobiological factors, psychiatric or medical comorbidities, or medication side effects.

The prior studies all used parent-report measures to examine associations between clinical indicators of psychopathology and observable behaviors and/or patient characteristics. A slightly different approach was taken by Handen et al. to examine risks for self-injurious behavior (SIB), comparing parent-reported SIB at admission and SIB observed by direct care staff in the inpatient setting. The authors found that while $74 \%$ of youth had parent-reported SIB at home, only $25 \%$ were observed to engage in SIB while hospitalized. Patients with SIB in both home and hospital had significantly lower non-verbal IQ, greater social communication deficits, more stereotyped behavior and higher scores on the Repetitive Behavior Scale Revised, SIB subscale (Handen et al. 2018). This paper provides vital information that may aid caregivers and clinicians in identifying SIB risk in children with ASD.

Finally, this special issue highlights the urgent need for new measurement tools to adequately capture important dimensions in youth with ASD across the full range of intellectual and verbal abilities. Specifically, a previous article by Mazefsky et al. describe development of the Emotion Dysregulation Inventory (EDI), a parent-report questionnaire designed to measure emotional distress and difficulty with emotion regulation in ASD, including in minimally verbal and intellectually disabled individuals (Mazefsky et al. 2016). The EDI was developed using guidelines from the Patient-Reported Outcomes Measurement Information System (PROMIS ${ }^{\circledR}$; National Institute of Health 2014) and tested with the AIC sample. The psychometric properties of the EDI are reported in the current special issue (Mazefsky et al. 2018). The EDI was sensitive to change from admission to discharge and was normally distributed across the range of verbal ability and non-verbal IQ. The EDI continues to be used, normative data are being collected in the AIC and other research cohorts, and the measure will likely become a valuable tool to better assess symptoms and outcomes across the spectrum.

\section{Considerations for Future Research}

The articles discussed demonstrate both the feasibility and challenges of inpatient research, and highlight the utility of focusing on those more severely affected by ASD to deepen our understanding of the disorder. The articles presented here point toward several future directions that would 
advance both scientific understanding and clinical prevention and intervention strategies.

Research on psychiatric hospitalization of children with ASD as an intervention is generally minimal, and is an area of opportunity as use of this intervention is common and costly (Croen et al. 2006). Access to the type of specialized inpatient units where the AIC is being conducted is very limited, leading some researchers to develop recommendations for best practices (McGuire et al. 2015) and clinical care pathways (Kuriakose et al. 2018) that seek to improve the care that youth with ASD and other developmental disabilities can access in the much more prevalent non-specialized psychiatric units. As a public policy matter, further research is needed to assess the specific treatment and environmental factors that lead to better outcomes in either specialized or general settings, and may engender comparative effectiveness research on the two settings. Future research is also needed to better understand durability of treatment, as Pedersen and colleagues showed some regression in some aspects of improvement post-discharge. Differences in prescribing practices of specialty inpatient providers versus community providers could be one important factor suggested by the study of Wink and colleagues, and merits more specific investigation of pharmacologic approaches to severe, refractory problems leading to psychiatric hospitalization.

Given the greater presence of depression and oppositional defiant disorder in verbal youth relative to those with minimal verbal ability, further intervention work to increase communication of emotional difficulties in verbal children with ASD is needed. However, as stated by the authors, lower rates of depression in minimally verbal youth may be biased due to self-report challenges, and development of tools to better identify emotional states in verbally challenged individuals is also needed. In addition, Williams et al. found that externalizing behavior severity was not associated with verbal ability, and that coping/adapting skills were most relevant. As the imminent onset of externalizing behaviors can be difficult to predict in those with limited verbal ability, and even the fluently verbal, use of objective measures, such as biosensors of physiology, could be useful in recognizing states of agitation and providing a window for implementation of coping/adapting strategies before the onset of challenging behavior (Williams et al. 2017).

The role of diagnostic overshadowing (Reiss and Szyszko 1983) was also highlighted when considering the presentation and prevalence of symptoms of trauma and suicidality in youth with ASD. Further research is needed to determine the sensitivity and specificity of clinical criteria for diagnosing disorders such as PTSD within this population. As stated by Brenner and colleagues, more subtle indicators such as distressing memories, intrusive thoughts, loss of interest, and lethargy should be examined in relation to possible on-going or historical abuse. The development of validated tools to evaluate suicidality is also an urgent need in ASD, highlighted by the paper of Horowitz and colleagues. Children with ASD can have difficulty with communication, abstract reasoning, identifying emotions, cause and effect, and also can have morbid or violent special interests. Due to these challenges, current suicide risk detection measures may not be valid for youth with ASD.

Increasing our understanding of SIB and the discrepancy between SIB observed inside and outside of the home is of great importance for the safety of children with ASD. While Handen and colleagues examined patient characteristics that might account for the discrepancy and help to distinguish between individuals who reportedly exhibit SIB in the home versus those who exhibit SIB in the home and hospital setting, parental and home factors were not measured. Future research accounting for parent and home setting variables could advance predictive models for identifying those most at-risk for SIB.

Finally, the example of Mazefsky et al. in carefully developing a measure using best practices that appears to be valid and normally distributed across the range of ASD deficits is a potent model for advancing measurement science in ASD. There remain multiple areas of behavioral and psychiatric functioning for which measures adequately validated for the full ASD population are lacking, including the common comorbidities of anxiety and depression, and this stands as a major barrier to developing effective treatments.

\section{Conclusion}

In summation, the articles in this special issue highlight the potential in studying populations enriched for the more severely affected end of the autism spectrum, the possibility of broadening the field's research settings to include inpatient and more naturalistic environments, and the opportunities likely to present from a large, accessible database of phenotypic and ultimately genetic data, using measures validated for individuals with ASD who are minimally verbal, intellectually challenged and/or have severe challenging behaviors.

Acknowledgments The author thanks Bryan King, MD, MBA, Briana Taylor, PhD and Mary Verdi, MA, for their assistance with this manuscript.

Author Contribution MS conceived and wrote the manuscript.

Funding The Autism Inpatient Collection (AIC) phenotypic database and biorepository is supported by a grant from the Simons Foundation Autism Research Initiative and the Nancy Lurie Marks Family Foundation (SFARI \#296318 to M.S.). 


\section{Compliance with Ethical Standards}

Conflict of interest The author declares no conflicts of interest.

Research Involving Human Participants All procedures performed involving human participants were in accordance with the ethical standards of the institutional research committees where the data was collected and with the 1964 Helsinki Declaration and its later amendments or comparable ethical standards.

Informed Consent Informed consent was obtained from all individual participants included in the study.

\section{References}

Aman, M. G., Singh, N. N., Stewart, A. W., \& Field, C. J. (1985). The aberrant behavior checklist: a behavior rating scale for the assessment of treatment effects. American Journal of Mental Deficiency, 89(5), 485-491.

Baghdadli, A., Pascal, C., Grisi, S., \& Aussilloux, C. (2003). Risk factors for self-injurious behaviours among 222 young children with autistic disorders. Journal of Intellectual Disability Research, 47(Pt 8), 622-627.

Bodfish, J. W., Symons, F. J., Parker, D. E., \& Lewis, M. H. (2000). Varieties of repetitive behavior in autism: comparisons to mental retardation. Journal of Autism and Developmental Disorders, 30(3), 237-243.

Brenner, J., Pan, Z., Mazefsky, C., Smith, K. A., \& Gabriels, R. (2017). Behavioral symptoms of reported abuse in children and adolescents with autism spectrum disorder in inpatient settings. Journal of Autism and Developmental Disorders. https://doi.org/10.1007/ s10803-017-3183-4.

Croen, L. A., Najjar, D. V., Ray, G. T., Lotspeich, L., \& Bernal, P. (2006). A comparison of health care utilization and costs of children with and without autism spectrum disorders in a large groupmodel health plan. Pediatrics, 118(4), e1203-e1211. https://doi. org/10.1542/peds.2006-0127.

Gadow, K. D. S. (2013). Child and Adolescent Symptom Inventory-5 (Ages 5 to 18 Years). Stony Brook: Checkmate Plus.

Hall-Lande, J., Hewitt, A., Mishra, S., Piescher, K., \& Laliberte, T. (2014). Involvement of children with autism spectrum disorder (ASD) in the child protection system. Focus on Autism and Other Developmental Disabilities, 30(4), 237-248.

Handen, B. L., Mazefsky, C. A., Gabriels, R. L., Pedersen, K. A., Wallace, M., \& Siegel, M. (2018). Risk factors for self-injurious behavior in an inpatient psychiatric sample of children with autism spectrum disorder: A naturalistic observation study. Journal of Autism and Developmental Disorders. https://doi.org/10.1007/ s10803-017-3460-2.

Horowitz, L. M., Thurm, A., Farmer, C., Mazefsky, C., Lanzillo, E., Bridge, J. A., et al. (2017). Talking about death or suicide: Prevalence and clinical correlates in youth with autism spectrum disorder in the psychiatric inpatient setting. Journal of Autism and Developmental Disorders. https://doi.org/10.1007/s1080 3-017-3180-7.

Kuriakose, S., Filton, B., Marr, M., Okparaeke, E., Cervantes, P., Siegel, M., et al. (2018). Does an autism spectrum disorder care pathway improve care for children and adolescents with ASD in inpatient psychiatric units? Journal of Autism and Developmental Disorders. https://doi.org/10.1007/s10803-018-3666-y.

Lam, K. S., \& Aman, M. G. (2007). The repetitive behavior scalerevised: Independent validation in individuals with autism spectrum disorders. Journal of Autism and Developmental Disorders, 37(5), 855-866. https://doi.org/10.1007/s10803-006-0213-z.

Lerner, M. D., Mazefsky, C. A., Weber, R. J., Transue, E., Siegel, M., \& Gadow, K. D. (2017). Verbal ability and psychiatric symptoms in clinically referred inpatient and outpatient youth with ASD. Journal of Autism and Developmental Disorders. https://doi. org/10.1007/s10803-017-3344-5.

Mandell, D. S. (2008). Psychiatric hospitalization among children with autism spectrum disorders. Journal of Autism and Developmental Disorders, 38(6), 1059-1065. https://doi.org/10.1007/s1080 3-007-0481-2.

Matson, J. L., Boisjoli, J., \& Mahan, S. (2009). The relation of communication and challenging behaviors in infants and toddlers with autism spectrum disorders. Journal of Developmental and Physical Disabilities, 21, 253-261.

Mazefsky, C. A., Day, T. N., Siegel, M., White, S. W., Yu, L., \& Pilkonis, P. A. (2016). Development of the emotion dysregulation inventory: A PROMIS(R)ing method for creating sensitive and unbiased questionnaires for autism spectrum disorder. Journal of Autism and Developmental Disorders. https://doi.org/10.1007/ s10803-016-2907-1.

Mazefsky, C. A., Yu, L., White, S. W., Siegel, M., \& Pilkonis, P. A. (2018). The emotion dysregulation inventory: Psychometric properties and item response theory calibration in an autism spectrum disorder sample. Autism Research, 11(6), 928-941. https://doi. org/10.1002/aur.1947.

McGuire, K., Erickson, C., Gabriels, R. L., Kaplan, D., Mazefsky, C., McGonigle, J., et al. (2015). Psychiatric hospitalization of children with autism or intellectual disability: consensus statements on best practices. Journal of the American Academy of Child and Adolescent Psychiatry, 54(12), 969-971. https://doi.org/10.1016/j. jaac.2015.08.017.

Pedersen, K. A., Santangelo, S. L., Gabriels, R. L., Righi, G., Erard, M., \& Siegel, M. (2017). Behavioral outcomes of specialized psychiatric hospitalization in the Autism Inpatient Collection (AIC): A multisite comparison. Journal of Autism and Developmental Disorders. https://doi.org/10.1007/s10803-017-3366-z.

Reiss, S., \& Szyszko, J. (1983). Diagnostic overshadowing and professional experience with mentally retarded persons. American Journal of Mental Deficiency, 87(4), 396-402.

Righi, G., Benevides, J., Mazefsky, C., Siegel, M., Sheinkopf, S. J., \& Morrow, E. M. (2017). Predictors of inpatient psychiatric hospitalization for children and adolescents with autism spectrum disorder. Journal of Autism and Developmental Disorders. https ://doi.org/10.1007/s10803-017-3154-9.

Rosenberg, R. E., Mandell, D. S., Farmer, J. E., Law, J. K., Marvin, A. R., \& Law, P. A. (2010). Psychotropic medication use among children with autism spectrum disorders enrolled in a national registry, 2007-2008. Journal of Autism and Developmental Disorders, 40(3), 342-351. https://doi.org/10.1007/s10803-009-0878-1.

Sannar, E. M., Palka, T., Beresford, C., Peura, C., Kaplan, D., Verdi, M., et al. (2017). Sleep problems and their relationship to maladaptive behavior severity in psychiatrically hospitalized children with autism spectrum disorder (ASD). Journal of Autism and Developmental Disorders. https://doi.org/10.1007/s1080 3-017-3362-3.

Siegel, M., Doyle, K., Chemelski, B., Payne, D., Ellsworth, B., Harmon, J., et al. (2012). Specialized inpatient psychiatry units for children with autism and developmental disorders: a United States survey. Journal of Autism and Developmental Disorders, 42(9), 1863-1869. https://doi.org/10.1007/s10803-011-1426-3.

Siegel, M., Smith, K. A., Mazefsky, C., Gabriels, R. L., Erickson, C., Kaplan, D., et al. (2015). The autism inpatient collection: methods and preliminary sample description. Molecular Autism, 6, 61 . https://doi.org/10.1186/s13229-015-0054-8. 
Williams, D. L., Siegel, M., \& Mazefsky, C. A. (2017). Problem behaviors in autism spectrum disorder: association with verbal ability and adapting/coping skills. Journal of Autism and Developmental Disorders. https://doi.org/10.1007/s10803-017-3179-0.

Wink, L. K., Pedapati, E. V., Adams, R., Erickson, C. A., Pedersen, K. A., Morrow, E. M., et al. (2017). Characterization of medication use in a multicenter sample of pediatric inpatients with autism spectrum disorder. Journal of Autism and Developmental Disorders. https://doi.org/10.1007/s10803-017-3153-x. 